\title{
MYCOsoft: A mycological database
}

\author{
Ajit Kumar Saxena ${ }^{1 \star}$, Jyoti Gupta ${ }^{1}$, S. Pandey ${ }^{1}$, A. N. Gangopadhyay ${ }^{2}$ and L. K. Pandey ${ }^{3}$ \\ ${ }^{1}$ Human Molecular Cytogenetic Laboratory, Centre of Experimental Medicine and Surgery, \\ Banaras Hindu University, Varanasi-221005, India. \\ ${ }^{2}$ Department of Obstetrics and Gynecology, Institute of Medical Sciences, Banaras Hindu University, \\ Varanasi-221005, India. \\ ${ }^{3}$ Department of Pediatric Surgery, Institute of Medical Sciences, Banaras Hindu University, Varanasi-221005, India.
}

Accepted 3 May, 2012

\begin{abstract}
The realization regarding the significance of microorganisms is increasing with the passage of time. In today's scenario of global climate change and increasing threats to biodiversity, the wheel of advanced biotechnologies is revolving around microbes to restore the ecological balance lost in the past few decades. In Pakistan, however, we lack sufficiently reliable biological data to start with and our knowledge of functional importance of the indigenous microbiota is highly fragmentary. Cataloging and preserving of our rich flora is therefore of extreme importance for regional and national resource management, bioprospecting and fundamental scientific research. Some institutes have their own collection but status of preservation is not satisfactory. The present project had helped to initiate the task to develop computerized data software for an easy access to the cumbersome process of identification. The software entitled "MYCOsoft" would be available in the market as well as from FCBP on request.
\end{abstract}

Key words: MYCOsoft, microbial diversity, biodiversity conservation, microbial germplasm.

\section{INTRODUCTION}

The importance of microorganisms in human affairs is well known. All the world's civilizations have used products derived from microorganisms; and human beings have been making microorganisms work for them for a very long time even before knowing that they exist. For example, Egyptians were the first to use yeast to produce leaven bread about 6000 years ago and the Chinese used moldy soybean curd as an antibiotic in 500 B.C. So, the application of microorganisms and their use in service of man are not new. When scientific explanations for these simple processes began to be established, culture collections became the basis of great industries and modern techniques have given us new and far-ranging powers over microorganisms.

The conservation of all kind of living organisms including the microbial gene pool, with the aim of improving human environment and welfare through prudent utilization of the full potentials of living resources,

\footnotetext{
${ }^{*}$ Corresponding author. E-mail: draksaxena1@rediffmail.com.
}

has been a subject of concern for United Nations Environment Program (UNEP) for quite some time.

In fact, modern biotechnology is enabling scientists to change and manipulate microorganisms in ways that seemed the stuff of science fiction just a few years ago. The developers of these new technologies are eager to see products that kill pests, clean up oil spills and toxic chemicals, fertilize crops, improve human and animal health, and much more. Achieving such a potential will depend on reliable culture collections and effective infrastructure for exploiting full potential of microorganisms. The success and potential of biotechnology relies on the diversity of microorganism and the biodiversity of the molecules they produce as a result of primary and secondary metabolism and on the conservation of genetic resources they provide. However, more than 30 years after Stockholm conference on human environment, we can say that the world community has been concerned for some time about the conservation of diversity in higher plants and vertebrates. Equal attention has not been given to microorganisms irrespective of their importance. There is a need to 
persuade policy-makers to be more concerned than they currently are about the conservation of diversity in microorganism.

There is a wide variation in the estimates of the number of species in the world. This is due to the lack of sufficiently reliable biological data and the fact that our knowledge of biology of most species is virtually nonexistent and our knowledge of knowledge of their functional importance of remains fragmentary. This is compounded by the fact that increasing levels of complexity and new habitats are discovered progressively. We cannot measure satisfactorily the world's microbial diversity and our estimates of the loss of microbial biodiversity are therefore conjectural. In the case of the fungal gene pool, the number of species of fungi currently maintained in culture collection throughout the world represents about $17 \%$ of the 80,000 accepted species of fungi, which itself is far short of the total number of fungal species that has long been quoted as 250,000 but is now conservatively estimated at 1.5 million. The same holds true for other groups (Hawksworth, 1995).

Completing the inventory of the world's biodata for microorganisms will clearly be a major task. Culture collections, their associated laboratories and biosystematists have a crucial role in efforts to respond to this problem. Biosystematics has declined; thus, there is a need to emphasize the contributions of taxonomy in successful applications of microorganisms to agriculture, medicine, and industry, in goods production and resource conservation. There is a need to establish internationally agreed priorities, develop identification services and training courses in taxonomy of fungi.

The present effort has therefore been made to convert the data regarding the microbes in to a bioinformatics software by the name of "MYCOsoft". This software would be available to the scientist and researchers enabling them to identify fungi independently. "MYCOsoft" would shorten the lengthy procedure of identification bringing the whole procedure with the reach of just one click without opening massive compendia and identification manuals.

\section{LITERATURE REVIEW}

There is an array of microorganisms like algae, bacteria, fungi, protists, and viruses, not normally visible to the naked eye, but are essential components of biological diversity, without which there can be no sustainable ecosystem (Hawksworth, 1991; Hawksworth, 1992; Hawksworth and Colwell 1992; Sly, 1994; Staley et al., 1997). $50 \%$ of the living biomass on the earth is microbial consortia and microorganisms provide a major source of genetic information for molecular biology and biotechnology (Bull et al., 1992; Nisbet, 1992). Under accelerating pressures of climate change and human perturbations, the natural habitats that harbor these undiscovered microorganisms are disappearing rapidly.

Destruction of tropical forests is estimated at between $16.4 \times 10^{6}$ and $20.4 \times 10^{6}$ hectares per year. Only a small percentage of the Earth's temperate forests remain. The amount and quality of the world's natural habitat are declining, and only the deepest parts of the ocean appear to have maintained some degree of pristine quality. Microorganisms, too, are disappearing from the Earth (Lean and Hinrichsen, 1992).

Standard policies, such as the US Endangered Species Act (ESA) and the Convention on International Trade in Endangered Species of Fauna and Flora (CITES) protect individual species but microorganisms, especially those we have not yet discovered, cannot be protected in this way. Habitat protection is a means to protect organisms and their habitats (Eisner et al., 1995), a method that would be more beneficial to protection of microbial biodiversity.

Hawksworth (1995) pointed out that completing worldwide inventory of biota requires additional taxonomic collections, training of people in many different countries to carry out taxonomic studies, and serious international coordination of these efforts. The African Network of Microbiological Resources Centres (MIRCENs) is a step in that direction (DaSilva, 1993). A new MIRCEN, focusing on microorganisms used in biohydrometallurgical processes has been established in Pune, India (Anon, 1995). Not only are culture collections important, but in a world united by electronic communication, computer databases may supply invaluable information to taxonomists and other researchers. A move to link several microbiological databases and add functional data to the genetic data was explored at the recent International Symposium on Microbial Ecology in Santos, Brazil (Wertheim, 1995). There are 521 culture collection centers working in 66 countries worldwide. Some worldwide known culture collection centres are International Mycological Institute (IMI), American Type Culture Collection (ATCC), Belgian Co-ordinated Collections of Micro-organisms (BCCM), Centraalbureau voor Schimmelcultures (CBS). IMI was founded in 1920 and is part of CAB International, an organization supported by 32 Member Governments established by treaty and with international legal status. The IMI culture collection comprises over 16,500 strains of filamentous fungi, yeasts and bacteria of interest in plant pathology, industry, biodeterioration studies, standards testing and specifications, systematic and biochemical research and education. Uses of fungi include biosynthesis of organic compounds, physiological assay, soil analysis, and enzyme production. The institute provides identification, preservation, microbial testing of materials, contract, consultancy, and training, development of preservation protocols, safe deposit and patent deposit services. ATCC was established in 1925 when a committee of scientists recognized a need for a 
central collection of microorganisms that would serve scientists all over the world. The early years were spent at the McCormick Institute in Chicago until the organization moved to Georgetown University in Washington, D.C., in 1937. As research in the biosciences expanded, ATCC began to diversify its holdings, and as the collections grew ATCC occupied a series of sites, each providing more storage space. ATCC moved to its current state-of-the-art laboratory in 1998. Common Access to Biological Resources and Information (CABRI) provides a unified search interface for a handful of European culture collections. These include BCCM, CABI and CBS, among others CBS is an important center for mycological research in The Netherlands. Their extensive culture collection can be searched on many criteria, including cultural characteristics. CBS also provide access to databases on fungal nomenclature.

\section{Status of microbial conservancy in Pakistan}

Until 3 years ago in Pakistan, few research groups (NIBGE and CEMB) were holding small collections. Most of the research was based on foreign microbial cultures. This was creating a huge economic burden also causing unnecessary delays and discontinuity of research due to accidental loss or death of cultures. Mycologists and plant pathologists have been culturing microorganisms specially fungi for a long time but there has been no maintenance of microbial cultures in Pakistan with the result that whenever cultures were needed by researchers, teachers and students, they were not available anywhere in Pakistan.

Under the newly established Department of Mycology and Plant Pathology, First Fungal Culture Bank of Pakistan (FCBP) was established early in 2003. From June 18,2003 , it started functioning with a meager staff, an Incharge with two research associates. FCBP is registered with the following international organizations: The World Data Centre for Microorganisms (WDCM), World Federation of Culture Collection (WFCC) and Microbial Research Centre (MIRCEN). The main activity of FCBP is isolation in pure culture, identification and preservation of fungi of Pakistan. It has already obtained pure culture, identified and preserved over 800 strains of fungi. On excessive demand of researchers for cultures of bacterial species, about six months ago, bank has also initiated isolation and conservation of bacteria as well. Now bank hold inventory of around 62 isolates of bacteria, this activity will also continue. FCBP is providing authentic cultures to researchers, teachers and students on nominal charges. Disease diagnostic services are also being provided.

Many researchers and research students isolate fungi and other microorganism but they cannot identify them. FCBP is providing facility to identify such cultures up to species level. It has already provided facility to various research organizations and universities all over Pakistan and activity is in progress. It offered internship on isolation, purification, preservation and identification of fungi during last one year since the internship program was started by Pakistani universities and also providing training to students of the Punjab University. FCBP is supervising M.Sc. (Hons.) and Ph.D. research in taxonomy to overcome scarcity of manpower in this field. It provides training to college teachers and researchers in isolation, identification and conservation of microbial flora. FCBP organized a week long workshop on "Identification and Conservation of Micromycetes" from August 23 to 28, 2004 with financial support of HEC. Submitted a program to HEC for holding second workshop and intends to make it an annual feature. MYCONEWS', an official publication of FCBP since June, 2003 has just completed its three years of publications (13 issues). MYCONEWS consists of news, views and research notes. The most important regular feature is a list of updated accessions of cultures of Pakistani fungi in each issue published as "Current Inventory of FCBP" and also disseminates inventory of bacterial isolates. FCBP research work is also published in the form of Research Bulletins. First research bulletin entitled "NEW RECORDS OF FUNGI IMPERFECTI FROM PAKISTAN" was published in April 2005. This research bulletin includes 22 species of fungi imperfecti as new records with descriptions and microphotographs. It includes five species of Aspergillus, four species of Phoma, two species of Acremonium, two species of Curvularia, two species of Fusarium, two species of Trichoderma and one species of Alternaria. Second research bulletin entitled "COPROPHILOUS FUNGI OF PAKISTAN" was published in December 2005. This research bulletin includes nineteen species of coprophilous fungi with descriptions and microphotographs. Four species of Absidia, one species of Drechslera, two species of Pilobolus , one species of Fusarium, two species of Acremonium, one species of Saccobolus, two species of Ascobolus, one species of Penicillium, one species of Phycomyces, one species of Isaria, one species of Syncephalastrum, on species of Doratomyces, one species of Cephaliophora. Third bulletin by name of "THE GENUS TRICHODERMA IN PAKISTAN" was published in 2009. We are preserving our authentic cultures at $4^{\circ} \mathrm{C}$ in refrigerator, in mineral oil and at $-20^{\circ} \mathrm{C}$. FCBP has introduced silica gel technique in preserving cultures. It requires regular reculturing. All these methods are laborious, time consuming and expensive, sometimes resulting in loss of culture viability. FCBP has recently acquired cryopreservation system, to facilitate long-term preservation, and is going to equip itself with cleanroom technology, bio-safety cabinets to follow international standards. A project under PSF-US linkage (83 M) has been submitted for further improvement of infrastructure on international standards and its upgradation into 
National Microbial Culture Centre (NMCC). At the same time, it was realized that the data so far collected must be digitized and in a form that it would be accessed easily by the scientists. This idea prompted to work for the development of MYCOsoft for which the proposal was submitted to the University of the Punjab in 2008.

\section{METHODOLOGY}

\section{Data analysis}

The data presented in the form of research bulletin \#2 entitled "Coprophilous Fungi of Pakistan" published by First fungal Culture Bank of Pakistan (FCBP) in the year 2005, was analyzed carefully. Care was taken to observe that all the taxonomic parameters were taken into account. This resource is being updated regularly.

\section{Selection of parameters and their hierarchical arrangement}

The parameters were then selected in order of descending priority or significance. It means that less important characters were taken at the end, while characters with increasing significance were towards the top portion of the list. The characters were arranged and then the units were discussed and finalized on the basis of which the entries would be made.

\section{Defining ranges or limitations}

Each and every character, no matter at what level of priority it was, was discussed at length separately and the extent or limitations were earmarked before the entry of data in terms of real figures or units. For example colony diameter may range from 0.5 to $5.0 \mathrm{~cm}$.

\section{Data uploading}

The elaborate and lengthy phase of data entry was carried out after defining the limits. The program was regularly checked by running during this phase of data entry also and any loophole or flaw was removed for a smooth working of the program. Cross check and double check was also done at times to facilitate the working of the program.

\section{Uploading of images (micrographs and line drawings)}

The images or line drawings to support the software database were also uploaded along with the dataset. The synchronous working of the two data bases was also ensured carefully.

\section{SOFTWARE SPECIFICATION}

\section{Overview}

Help to develop the software was taken from IT officials of University of the Punjab.

\section{Scope of project}

The system consists of the following modules/features:

\section{Features}

The features are as follows:

1. Species group (up to 5 level).

2. Species parameters.

3. Species images.

4. Advance search (using Boolean operator).

\section{Backup and security}

1. Username/password protected.

2. Multi client supported.

3. Database backup feature.

\section{Requirements}

1. SQL Server 2000.

2. Dot Net Framework 2.0.

3. Microsoft XP Operating system or higher.

\section{Implementation}

1. Institute of Agricultural Sciences.

\section{Technical details}

\section{Application architecture}

Three-tier architecture is used in the development of our application and our application is both web and desktop based. The block diagram of three-tier architecture of our application is given in Figure 1.

\section{Software specification}

The following software should be installed:

1. Microsoft XP or Higher.

2. Dot NET Framework.

3. SQL Server 2000.

Tools and technologies: We used the Dot NET framework in the development of this project because our platform for running the software was also Microsoft. Another reason for using the Dot NET framework is that our application is multilingual, so we used localization feature provided by Microsoft Dot Net Framework. Using the feature of localization, our application can support more than 250 languages. It works efficiently on the Microsoft Windows.

SQL server: SQL server is one of the best options to develop a large database. So we used it at the back end of our project.

\section{Context level diagram}

The context level diagram is shown in Figure 2.

\section{Future plan}

The future plans are place all functionality of the system online, so that users can add/update/search specious information online. 

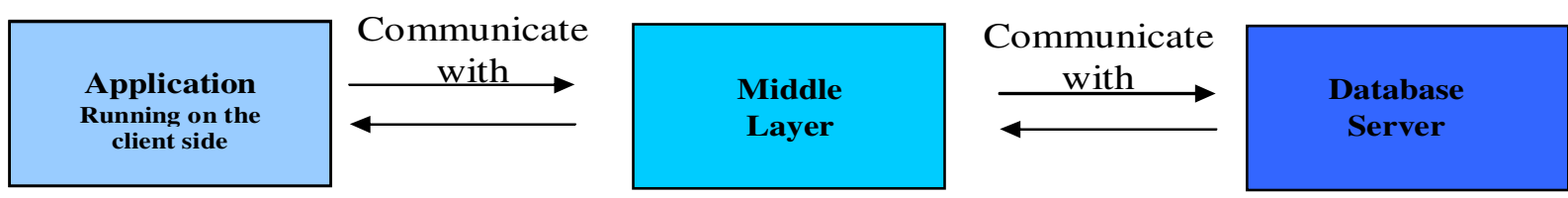

Figure 1. Block diagram of three-tier architecture.

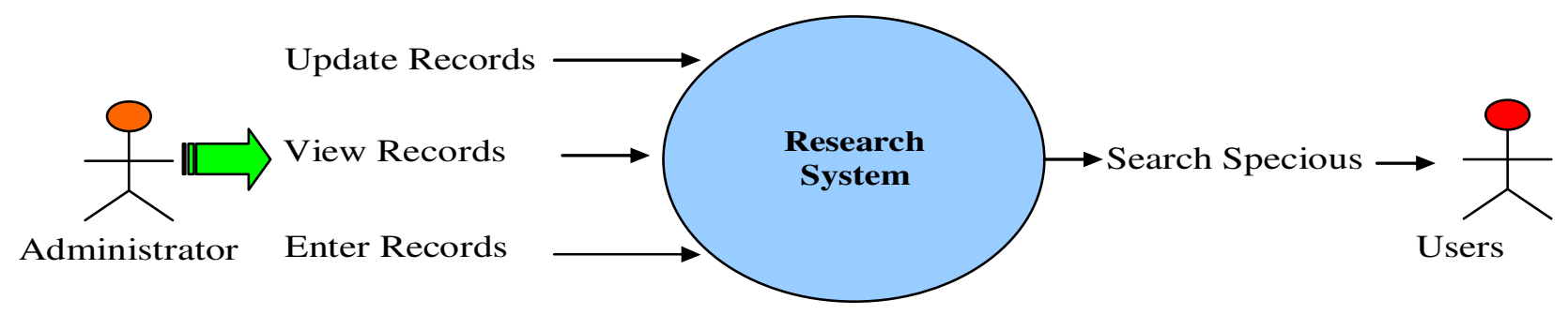

Figure 2. Context level diagram.

\section{Requirements}

\section{Hardware required}

1. One server machine with Microsoft Windows Server Operating System. This is a basic requirement.

2. Clients machines with Microsoft Operating System. This is a basic requirement.

3. Clients machines should be connected to server through LAN or internet.

4. Scanner for scanning Images.

\section{Software requirements}

1. Dot Net Framework 2.0 for client machines.

2. Microsoft Office should be installed on client machine for importing reports.

3. SQL Server 2005 on server machine.

\section{Operators}

\section{Training}

1. A technical person required to maintain database and able to install the application.

2. Data entry operator: It depends on the collection of specious.

\section{RESULTS}

\section{User manual}

The first screen is the splash screen followed by login screen and the main screen (Figure 3).

\section{Defining new group}

This is shown in Figure 4.

\section{Defining characteristics}

This is shown in Figure 5.

\section{Defining specious}

This is shown in Figure 6.

\section{Advance search}

This is shown in Figure 7.

\section{Change password}

This is shown in Figure 8.

\section{Backup data base}

Finally, we have a backup data base.

\section{DISCUSSION}

The climate of the planet Earth is changing rapidly and this change is creating pressure on the native fungal flora. A number of species are at the verge of extinction due to changing environmental conditions and habitat destruction. Biodiversity is going to face consequences which are irreversible and cascading. The pattern of species distribution is also changing due to such perturbations. As a result of which the organisms are pushed towards extinction while on the other hand intrusions into new geographical areas are also being 


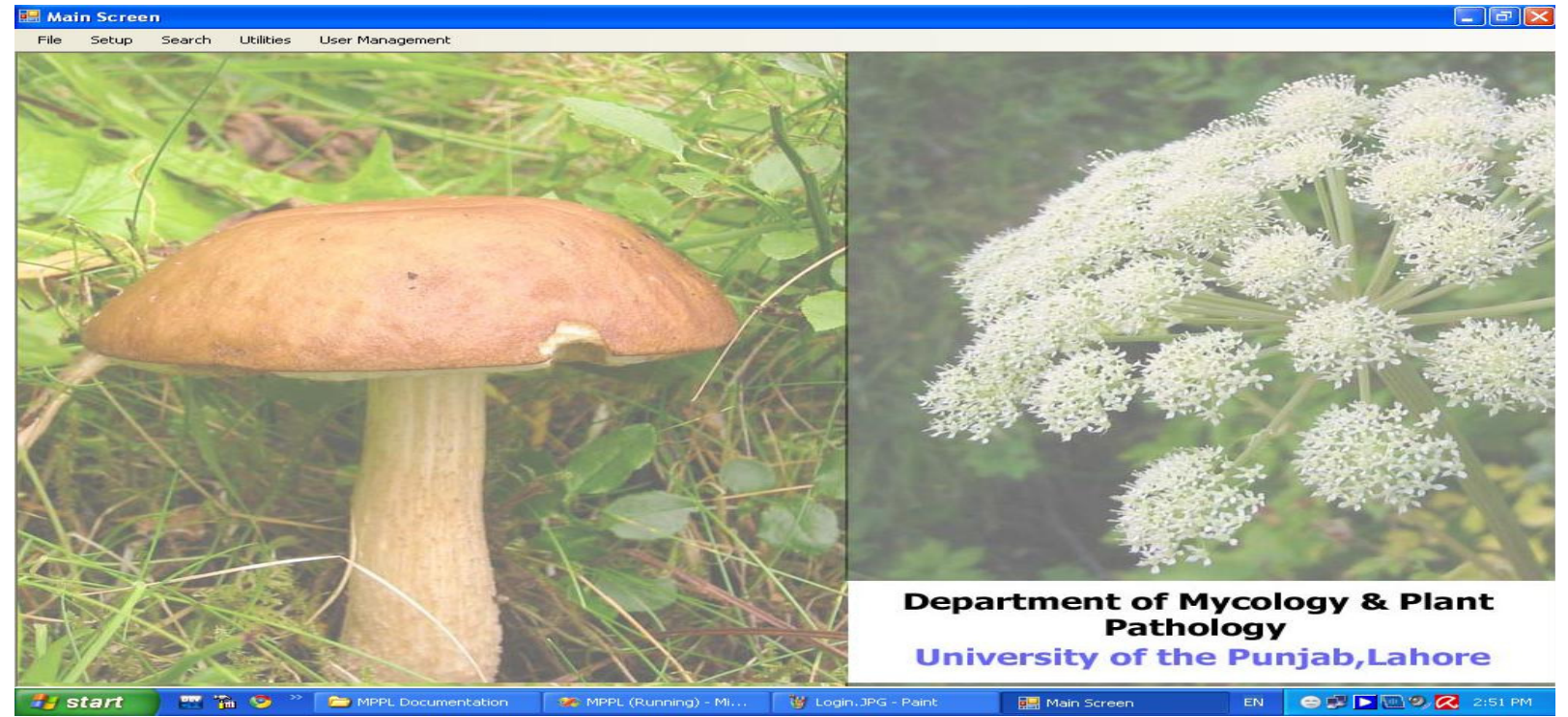

Figure 3. Splash screen.

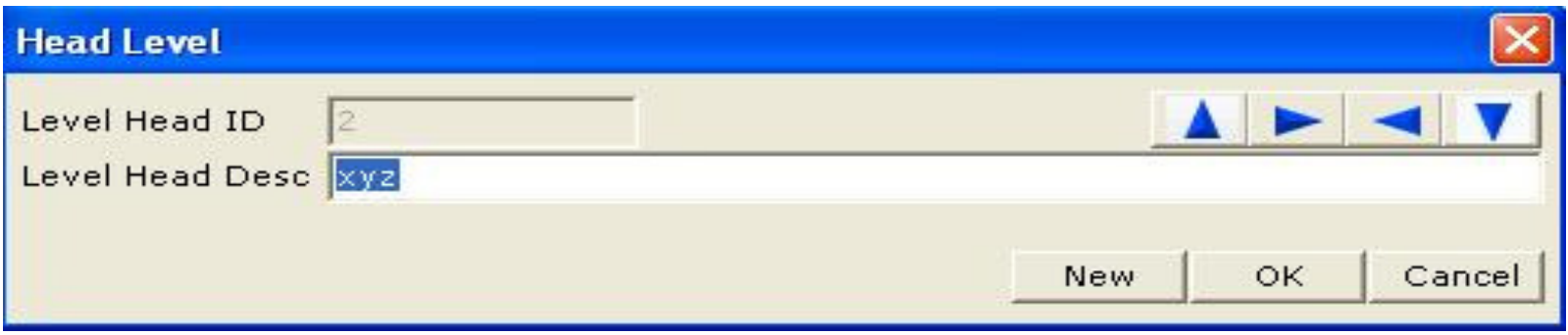

Figure 4. Defining new group.

\begin{tabular}{|c|c|c|}
\hline Parameter List & & 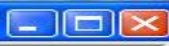 \\
\hline Parameter & Comments & I \\
\hline $\begin{array}{l}\text { artificial substrate } \\
\text { collumellae color } \\
\text { collumellae morphology } \\
\text { Colony Color } \\
\text { colony size } \\
\text { growth temperature } \\
\text { natural substrate } \\
\text { sporangia. } \\
\text { sporangia size } \\
\text { sporangiophore colour } \\
\text { sporangiophore morphology } \\
\text { sporangiophore size } \\
\text { sporangiospore color } \\
\text { sporangiospores shape } \\
\text { sporangiospores size }\end{array}$ & 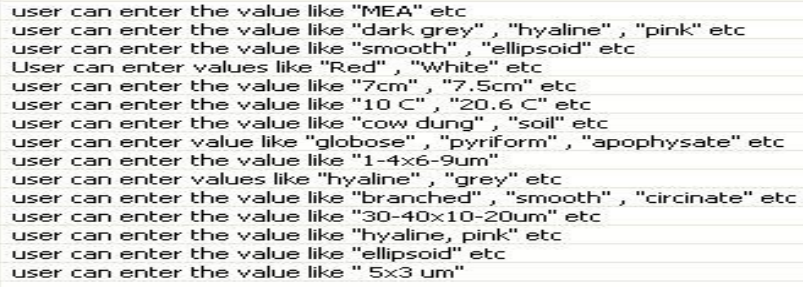 & \\
\hline & & \\
\hline & & \\
\hline & & \\
\hline & & \\
\hline Characteristic Desc & & \\
\hline Camments & & \\
\hline & OK & Cancel \\
\hline
\end{tabular}

Figure 5. Defining characteristics. 


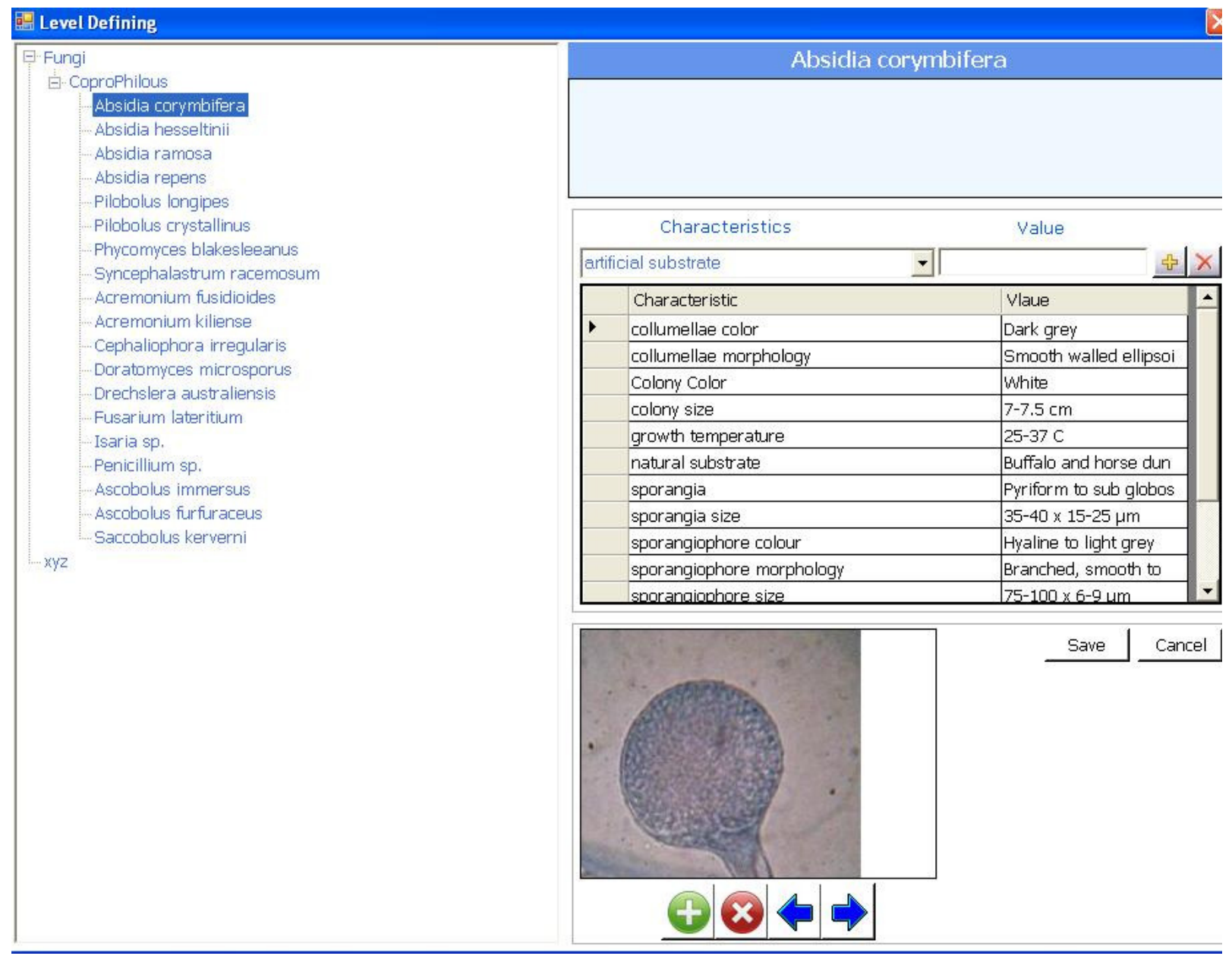

Figure 6. Defining specious.

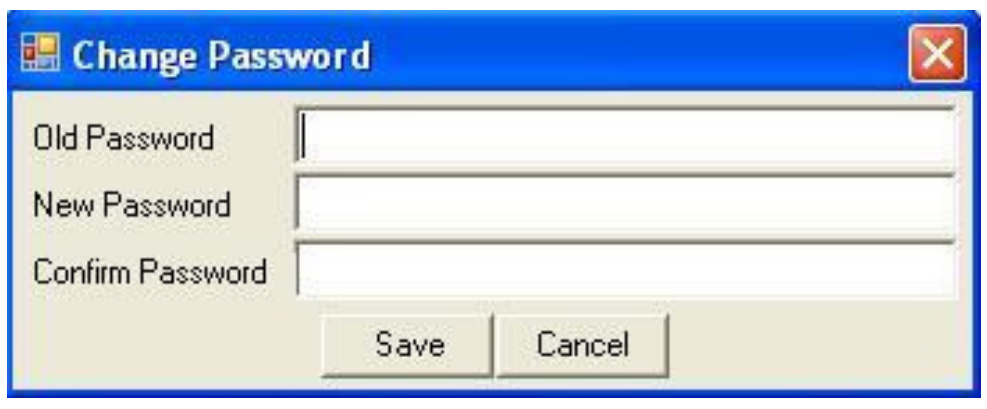

Figure 7. Changing password.

reported, so it is very important to have the electronic records of the native fungal flora. First, Fungal Culture Bank of Pakistan (FCBP) which was established in 2004 is working as a sole source of microbial collection and information. So, it was necessary to digitize the information of microbial collection at FCBP.

No doubt that the fungi can be identifying by growing them on culture media and recording characters like morphology and colour of the colony and colour, size and microscopic structures of the fungal propagules. Some of the books of fungal taxonomy also offer diagnostic keys to provide help in the process of identification. More importantly, one has to be a good taxonomist to reach to a subtle conclusion or at least rely on an expert fungal systematist. However, all these methods are time consuming and labour intensive. Therefore, it was the 


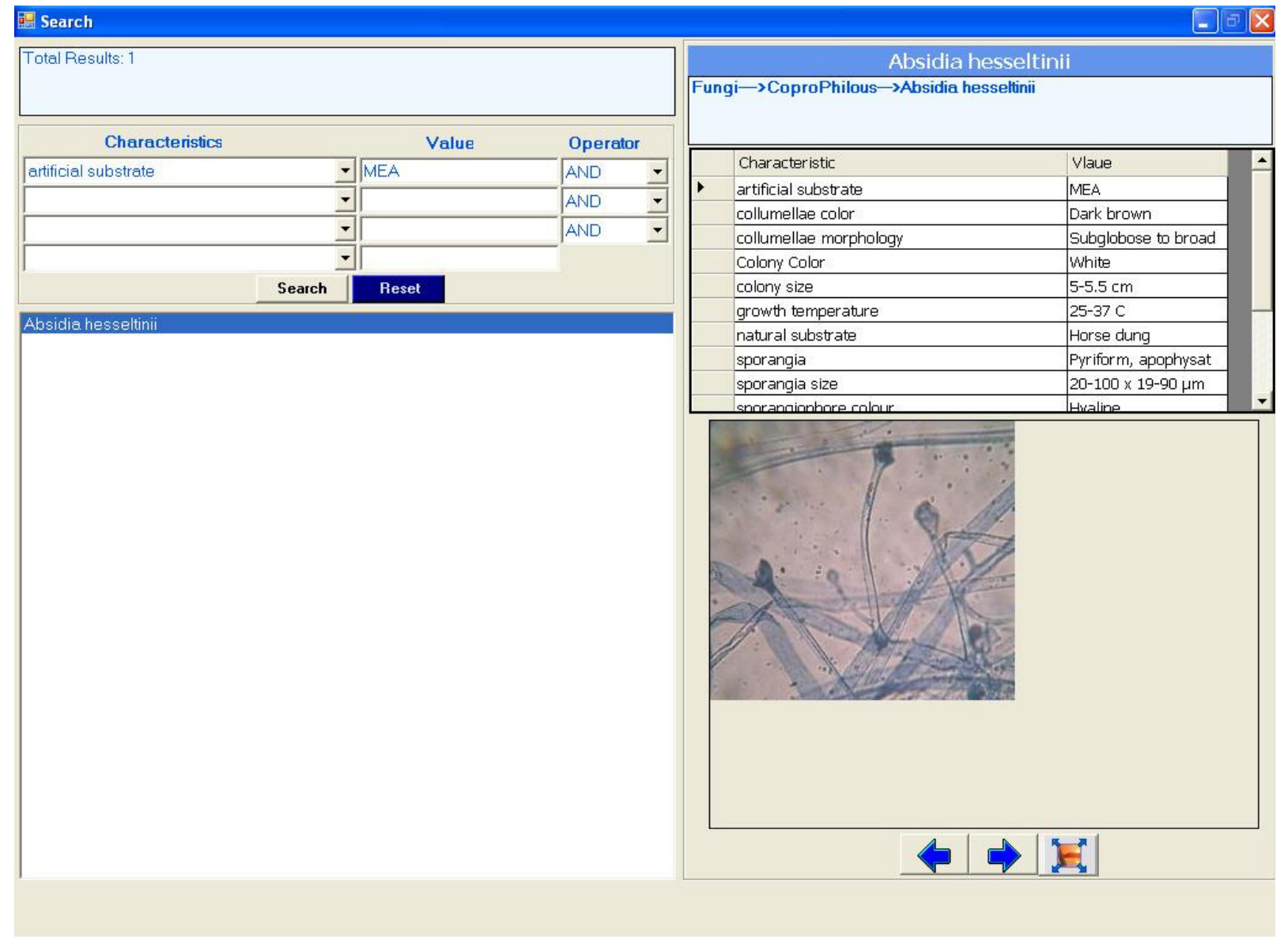

Figure 8. Advance search.

need of the hour to keep the knowledge updated and more reliable for the researchers in the recent scenario of global climate change. So, it was merely important to develop this software to make the way of identification easier and more authentic. It was developed for the cataloging of various groups of fungi. There is need to keep the pace with the world of information technology; so, it is a basic step to give the sense of bioinformatics in mycological studies.

\section{Main features of the software}

The following tasks are fulfilled by the software which has been developed by the Institute of Mycology and Plant Pathology in collaboration with a team of IT experts:

1. The electronic record of the fungal collection of FCBP would be maintained.

2. Can identify the specimen used by feeding simple characters.

3. The data is available on CD and online.

4. Others groups of fungi, bacteria, nematodes would be transformed into the same shape.
5. The software is multipurpose and has no limitation of making further entries or improvements.

6. Software requires a password for its operating, this is essential for the protection of the secured data.

7. It has the option to make a data file for a specific organism.

8. We can upload micro and macro features of the fungi additionally with their photographs. The remote users get the updates online or through new versions of MYCOsoft.

9. Software is able to complete search for a particular organism, if we provide just part of the name as key word.

10. Software is also able to do advanced search which is an important tool of it.

11. By the use of this software, we are able to digitalize our herbarium while cataloging the specimen.

\section{Activities done}

The following activities were done for the completion of the project:

1. Software development by using languages like $\mathrm{C}^{++}$and 
Oracle.

2. Data regarding characterization of the fungi was uploaded.

3. Initially the software is able to identify Coprophillous (growing on dung) fungi.

4. Data was equipped with macro, micro characters as well as their microphotographs.

5. CDs were also prepared for the dissemination and further presentation of the software.

6 . There is an option to upload further fungal groups.

\section{REFERENCES}

Anon (1995). Pune Mircen, World J. Microbiol. Biotechnol. IUMS 11(3):4-6.

Bull AT, Goodfellow M, Slater JH, (1992). Biodiversity as a source of innovation in biotechnology. Ann. Rev. Microbiol. 46:219-252.

DaSilva E (1993). African Network of Microbiological Resources Centres [MIRCENS]. Biofertilizer Production and Use. UNESCO, UNDP, Paris.

Eisner TJ, Lubchenco EO, Wilson DS, Bean MJ (1995). Building a scientifically sound policy for protecting endangered species. Science 268:1231-1232.

Hawksworth DL (1991). The Biodiversity of Microorganisms and Invertebrates: Its Role in Sustainable Agriculture. CAB International, UK.
Hawksworth D, Colwell R (1992). Biodiversity amongst microorganisms and its relevance. Biodivers. Conserv. 1:219.

Hawksworth DL (1995). The Convention on Biological Diversity: First Conference of the Parties [COP1]. World J. Microbiol. Biotech. IUMS $11[3]: 1-2$.

Hawksworth DL (1992). Biodiversity in microorganisms and its role in ecosystem function. IUBS Monograph 8, International Union. Biol. Sci. Paris, pp. 83-93

Lean G, Hinrichsen D (1992). WWF Atlas of the Environment. 2nd edn Harper Perennial, New York, p. 192.

Nisbet LJ (1992). Useful functions of microbial metabolites. CIBAFoundation Symp. 171:215-225.

Sly L (1994). Culture collections world-wide. In Kirsop, B. and Hawksworth, D. L. (Eds.). The biodiversity of microorganisms and the role of microbial resource centres. World Federation for Culture Collections. pp. 29-35.

Staley JT, Castenholz RW, Colwell RR, Holt JG, Kane MD, Pace NR, Salyers AA, Tiedje JM (1997). The Microbial World: Foundation of the biosphere. American Academy of Microbiology.

Wertheim M (1995). Call to desegregate microbial databases. Science 269:15-16. 